\title{
Desarrollo sostenible, humano y endógeno
}

\author{
Ignasi Brunet Icart \\ Rafael Böcker Zavaro
}

\section{Resumen}

Este artículo tiene por objetivo analizar la dispersión de las conceptualizaciones del desarrollo vinculadas a la "Segunda Década del Desarrollo". Dispersión que ha tenido lugar en el contexto de una economía basada en el conocimiento, modelada por el aprendizaje y motorizada por la innovación, y bajo el dominio o hegemonía de la economía neoclásica, que marca el capitalismo globalizado y financiarizado de las últimas décadas del siglo XX e inicios del siglo XXI. Hegemonía que obedeció en gran medida al descrédito del análisis keynesiano, a la crisis del estructuralismo latinoamericano y a la decadencia de las perspectivas críticas - neomarxistasdel desarrollo.

Palabras clave: desarrollo, derechos humanos, sostenibilidad, endógeno.

\section{Abstract}

\section{Sustainable development, human and endogenous}

This article aims to analyze the dispersion of the conceptualizations of development linked to the "Second Development Decade". This dispersion took place within a context of knowledge-based economy, which is shaped by learning and powered by innovation. A context dominated by neoclassical economics, which marked the globalized and financial capitalism of the late twentieth century and the early twenty first century. This neoclassical hegemony results from Keynesian analysis' discredit, the Latin-American structuralism crisis and the decadence of the critical views - development neo-Marxists.

Key words: development, human rights, sustainability, endogenous. 


\section{Introducción}

Mientras que la "Primera Década del Desarrollo" definió el desarrollo como un fenómeno económico que generaba y mantenía un incremento anual de Producto Nacional Bruto de 5\% a 7\% o mayor (Todaro, 1982; Rist, 2002), la Asamblea General de Naciones, al proclamar en 1970 la "Segunda Década del Desarrollo", planteó el desarrollo en términos de reducción de la pobreza, la desigualdad y el desempleo, dentro de un contexto de crecimiento económico. Novedad que se explica dentro de un proyecto de Naciones Unidas que debía integrar plenamente los componentes económicos y sociales en la formulación de políticas y programas para el desarrollo. Integración que ya inició la $11^{\text {a }}$ Conferencia Mundial de la Sociedad Internacional para el Desarrollo (SID) celebrada en Nueva Delhi (1969). Con la proclamación de la "Segunda Década", comenzó la búsqueda de un enfoque unificado del desarrollo; sin embargo, fue un proyecto frustrante y de corta vida, como empresa de Naciones Unidas. Ahora bien, el proyecto incubó la mayor parte de las ideas y lemas que animaron el debate sobre el desarrollo de las siguientes décadas, bajo la hegemonía de la teoría neoclásica del desarrollo.

Históricamente, la hegemonía de la economía neoclásica obedeció en gran medida al descrédito del análisis keynesiano, a la crisis del estructuralismo latinoamericano y a la decadencia de las perspectivas críticas - neomarxistas - del desarrollo, y en las que el subdesarrollo era una consecuencia del proceso continuo de extracción del excedente generado en la periferia por parte de los monopolios industriales del centro. Descrédito que explica la influencia del pensamiento neoclásico en las ideas y los programas de los principales organismos internacionales cuyas críticas al intervencionismo gubernamental y el culto a las virtudes del libre mercado procuraron una redefinición del Estado en todos sus frentes, tal y como quedó reflejado en la década de los ochenta con las denominadas políticas de estabilización seguidas de una política de ajuste estructural, y en la década de los noventa en el llamado Consenso de Washington.

En el marco de esta hegemonía, emerge un cuadro teórico sobre el desarrollo en el que se constata una dispersión de estrategias de desarrollo. Dicha dispersión está sustentada en la necesidad de vincular el desarrollo a la sostenibilidad y a la dimensión humana de la vida, y conectando esta dimensión a las prácticas cotidianas y al uso de los recursos y conocimientos locales y regionales como eje del desarrollo endógeno. Estas estrategias son las siguientes: desarrollo sostenible, desarrollo humano y desarrollo regional endógeno. Estrategias que forman parte de los procesos de reestructuración y recomposición espacial del capitalismo que han impulsado, para unos 
autores, la sociedad industrial, con la hegemonía del capital financiero en la escala global (Jameson, 2004; Mézáros, 2002), y, para otros autores, el desarrollo del posfordismo, esto es, de una economía global intensiva en conocimiento y su insumo estratégico: la innovación tecnológica (OCDE, 2001; Jessop y Sum, 2005; Castells, 2001). A través de la innovación, como factor fundamental en las dinámicas de desarrollo económico y empresarial, se ha generado la expresión "economía basada en la innovación", que describe las tendencias de las economías, afectadas por el ritmo de los avances científico-tecnológicos, su creciente complejidad y su papel determinante en los engranajes de la globalización económica posfordista, hacia una mayor dependencia del conocimiento, de la información, y de un mayor nivel de cualificación de los trabajadores (Arocena y Sutz, 2000; Fernández, Amin y Vigil, 2008).

El artículo tiene por objetivo exponer la visión del desarrollo vinculada a la "Segunda Década", que constituye la matriz tanto teórica como histórica de las distintas conceptualizaciones sobre el desarrollo. En primer lugar, se analiza la perspectiva del desarrollo sostenible. Esta plural estrategia de desarrollo arranca de la constatación del deterioro que el proceso productivo inflige en su entorno físico y social; deterioro que se constató a partir de la segunda mitad del siglo XX, y que suscitó controversias por la constatación de una previsible catástrofe ecológica. En segundo lugar, se plantea la perspectiva del desarrollo humano asociado al enfoque de los derechos humanos. Desde este enfoque, y desde la perspectiva de la justicia como imparcialidad (Rawls, 1996; Nussbaum, 1998), se evalúa el desarrollo y la equidad en un espacio más amplio que el de los ingresos, un espacio que no es sólo la propia satisfacción o utilidad, ni sólo la existencia de reglas iguales y comunes, sino el de las posibilidades para elegir y obtener la vida que tenemos; razones para valorar. En tercer lugar, se expone la perspectiva del desarrollo endógeno. Este enfoque surgió, a partir de la década de 1980, con la creciente dominación de la producción flexible, y apoyándose en la confluencia de dos líneas de investigación: 1) una de carácter teórico-empírico, que surge de la interpretación de los procesos de desarrollo industrial en determinadas localidades y regiones de Europa. Concretamente, de la llamada "tercera Italia" en la parte central-norte de Italia, del Estado de Baden-Wurtemberg en Alemania, y de la zona occidental de Flandes en Bélgica y; 2) otra de carácter teórico-político, que nace del intento de encontrar una noción de desarrollo que permitiera la acción pública para el desarrollo de localidades y regiones. 


\section{Desarrollo sostenible}

Fue el Club de Roma, en 1972, quien encargó un proyecto de estudio para analizar las causas y consecuencias a largo plazo del crecimiento de la población, el capital industrial, la producción de alimentos, el consumo de recursos y la contaminación. El informe final dio lugar a un libro, Los límites del crecimiento (Meadows, 1972), que llamó a la conciencia entre las élites y la propia opinión pública sobre las fatales consecuencias, en términos de agotamiento de recursos y deterioros humanos y medioambientales, que padecería el planeta en caso de mantenerse los sistemas de crecimiento, el modo de producción y el estilo de vida predominante en los países industrialmente desarrollados. Dos años más tarde, se publicó el segundo informe del Club de Roma, bajo el título La humanidad en la encrucijada, que regionalizó el primer informe, considerando las grandes regiones mundiales existentes. Ambos informes son los primeros, por otra parte, que hablan del surgimiento de un "sistema global" en el cual todas las naciones dependen de todas, y todas ellas del ecosistema.

Con posterioridad, en un informe de la World Conservation Union (1980) se utilizó por primera vez el concepto de desarrollo sostenible, y se lo definió teniendo como referencia central el espacio medioambiental. Para este informe es sostenible aquel desarrollo que respeta los ecosistemas, al tiempo se reconoce que, puesto que gran parte del crecimiento económico no genera una mejora del bienestar, no hay razón para pensar que el respeto medioambiental tenga que derivar en una pérdida de calidad de vida, sino todo lo contrario. Pero la confirmación de la crisis ecológica fue llevada a cabo por la Comisión Mundial sobre el Medio Ambiente y el Desarrollo de las Naciones Unidas en 1987. Para esta Comisión, el desarrollo sostenible es la forma de superar el conflicto entre los objetivos de conservación del medio ambiente y los de crecimiento económico que se habían presentado en su forma más cruda en el primer informe del Club de Roma Los límites del crecimiento. Este primer informe fue revisado veinte años después, confirmándose las previsiones del anterior aunque agravadas por las dos décadas transcurridas.

En el informe Brundtland de 1989 se acuñó la definición más comúnmente utilizada del concepto de desarrollo sostenible, al entender que el modelo de crecimiento imperante es insostenible por el planeta y amenaza la disponibilidad por parte de las generaciones venideras de determinados recursos no renovables que en el momento presente son consumidos sin límite alguno. Con el concepto de desarrollo sostenible se quiso, así, lanzar un proyecto político-económico-cultural de amplio alcance que fuese capaz 
de hacer coherentes las exigencias ambientales con las exigencias del desarrollo económico, desde un punto de vista de largo plazo. Se pretendía que los intereses de las generaciones futuras han de ser puestos explícitamente en primer plano junto con los intereses de las generaciones presentes, y se restringen los procesos de optimización económica al respeto de los límites impuestos por la reproducción de la biosfera.

La Conferencia de la ONU de Río de 1992 incorporará al concepto de sostenibilidad del Informe Brundtland el objetivo del desarrollo de los grupos más desfavorecidos de la Tierra. Incluso, tras la Cumbre Mundial de la Tierra de Johannesburgo, celebrada en 2002, se da cierto consenso en que la prioridad es la lucha contra la pobreza, y que se refleja de forma prístina en los ocho Objetivos de Desarrollo del Milenio, de la ONU, donde tan sólo en uno de ellos, el séptimo, se garantiza la sostenibilidad del medio ambiente. Un punto que, además, básicamente se refiere a cuestiones medioambientales relacionadas con la pobreza (reducir a la mitad el porcentaje de personas que carecen de acceso al agua potable, y mejorar considerablemente la vida de por lo menos 100 millones de habitantes de infraviviendas para el año 2020). De hecho, a partir del informe Brundtland y de la Conferencia de la ONU de Río existe una aceptación generalizada de la presencia de una auténtica crisis ecológica, y que exige un cambio sistémico y de la autocomprensión de la era industrial, por sus efectos desequilibradores que ha generado en el medio: contaminación, desaparición de especies, agotamiento de recursos, desertificación, degradación ambiental, etc. Sin embargo, en su lugar, "lo que hemos tenido en el último tercio del siglo XX, por parte de los grandes agentes de poder - las burocracias empresariales y estatales; la clase dominante que acapara recursos económicos e informativos - fue, en una primera fase, de negación de la realidad, y a partir de los años noventa maquillaje y juego de palabras" (Sempere y Riechmann, 2000: 262).

Al plantearse el desarrollo sostenible en los ámbitos económico, social y ambiental, resulta que todos, "desde el empresario centrado en la maximización del beneficio hasta el agricultor de subsistencia minimizador del riesgo, pasando por el trabajador social preocupado por la igualdad, el ciudadano amante de la vida salvaje o preocupado por la contaminación, el técnico maximizador del crecimiento, el burócrata centrado en la consecución de objetivos y por lo tanto el político preocupado por los votos" (Lélé, 1991: 613), están a favor del desarrollo sostenible (que es como estar a favor de la paz mundial), vaciándolo también, en parte, de contenido. Como expone Naredo, a las limitaciones que la escasez de recursos y el exceso de residuos ponían a la viabilidad del crecimiento ilimitado, "se respondió reforzando la meta del desarrollo con el calificativo de sostenible". Es decir, se respondió 
por el camino de buscar términos "cuyo carácter sintético facilite el consenso y soslaye o dé por superados los conflictos originarios. Se consiguió así un discurso único, blindado contra cualquier otro que trate de poner en cuestión su significado". A la vez que "por su capacidad para asimilar términos distintos a los suyos, ofrece una imagen de tolerancia que oculta el verdadero carácter represivo y autoritario que se deriva de su unidad. La nueva meta del desarrollo sostenible vino así a camuflar el antiguo conflicto entre desarrollistas y conservacionistas, logrando el consenso formal entre ambos sin necesidad de cuestionar la mitología originaria del desarrollo". Lo cual "propició un nuevo desarrollismo ahora a menudo calificado de 'ecológico' y/o 'sostenible"” (Naredo, 2006: 182).

Un nuevo "desarrollismo" en un contexto en el que, por un lado, los desequilibrios ecológicos adquieren una escala global y, por otro, el deterioro medioambiental del Norte, consecuencia del bienestar, encuentra su réplica cada vez en mayor medida en la degradación ecológica del Sur causada por la pobreza. De ahí que se ponga cada vez más en entredicho la noción convencional de desarrollo y de progreso y se imponga la convicción de que el modelo de desarrollo occidental, basado en técnicas pesadas muy agresivas para el medio natural y muy consumidoras de energías fósiles, no se puede generalizar a todos los países del mundo, máxime teniendo en cuenta las constricciones estructurales que para las acciones y los proyectos humanos se derivan de la finitud y vulnerabilidad de la biosfera, del carácter entrópico del universo y del aumento ineluctable de la población previsto para el próximo siglo.

El debate sobre el desarrollo sostenible, "por nuestro futuro común", tal y como prescribió la Comisión Brundtland, ha dado pie a distintas propuestas teóricas o estrategias de desarrollo sostenible. Una clasificación de dichas propuestas es la elaborada por Camagni (2005), distinguiendo dos dimensiones. La primera dimensión implica una primera dicotomía entre definiciones orientadas a los inputs de los procesos de producción y de intercambio, y definiciones orientadas a los outputs de los mismos. Tenemos, por un lado, definiciones que se basan en la necesidad de poner límites al uso de determinados recursos en el proceso de desarrollo económico, en el sentido de no exceder su capacidad de regeneración o su capacidad de asimilación. Por otro, tenemos definiciones que se basan en la necesidad de garantizar un flujo continuo de bienestar a largo plazo. En esta segunda aproximación se da una conceptualización débil de sostenibilidad, entendida en el sentido de que se permite una sustituibilidad más o menos amplia entre distintos elementos de la función de utilidad o de la función de producción. En la primera aproximación está, en cambio, implícita una idea de sostenibilidad 
fuerte, en la que ninguna reducción en la disponibilidad de un recurso no renovable puede ser compensada por un aumento en algún otro.

La segunda dimensión implica también una dicotomía según el tipo de racionalidad implícita. Así, se observan propuestas basadas en lo que ha sido denominado racionalidad formal o instrumental, definida como una racionalidad que supone la posibilidad de comportamientos siempre apropiados para alcanzar objetivos concretos en presencia de restricciones definidas. Se trata de una racionalidad que está detrás de gran parte de la elaboración económica neoclásica, que implica el supuesto de información perfecta, de conocimiento perfecto de las restricciones y de los resultados de las decisiones, de capacidad de cálculo perfecto e ilimitado. Las otras propuestas se basan en un distinto tipo de racionalidad, que se apoya en el análisis de procesos cognitivos más realistas en situaciones caracterizadas por información imperfecta, incertidumbre y complejidad. Una racionalidad procesual, definida no tanto a partir de la coherencia objetivos-medios-elecciones, sino sobre la corrección de un razonamiento y de un proceso de recogida y elaboración de la información. Pero Camagni destaca otra dimensión distinta y, por tanto, una nueva posible dicotomía, a través de la cual clasificar las definiciones y aproximaciones a la sostenibilidad. Esta nueva dimensión hace referencia a aquellas aproximaciones que se fundamentan en comportamientos económicos de mercado y aproximaciones que implican una ruptura neta con la organización institucional existente y la referencia a una nueva ética. Esta dimensión no es utilizada por este autor al considerarla errónea e inútilmente dicotómica ya que sólo hay una vía posible, la de un mercado orientado por una ética compartida. Pues no se trata de inventarse nuevas definiciones de mercado y de mecanismo económico, sino de aceptar que el mercado como institución social actúa y vive en el interior de normas, criterios, definiciones, valores definidos por el hombre; no se forma espontáneamente o naturalmente, sino que requiere, para poder funcionar, una adecuada cultura por parte de los actores implicados.

Otra clasificación es la elaborada por Castells (2001), cuando al analizar el ecologismo, como una nueva forma de movimiento social, procede a una distinción y a una tipología. Respecto a la distinción, Castells establece que hay que distinguir entre el medioambientalismo y la ecología. El medioambientalismo se refiere a todas las formas de conducta colectiva que, en su discurso y práctica, aspiran a corregir las formas de relación destructiva entre la acción humana y su entorno natural, en oposición a la lógica estructural e institucional dominante. La ecología se refiere a una serie de creencias, teorías y proyectos que consideran a la humanidad como un componente de un ecosistema más amplio y desean mantener el equilibrio del sistema en una 
perspectiva dinámica y evolucionista. El sentido del desafío ecologista es, para Daly (1991), la lucha por la redefinición histórica de las dos expresiones materiales fundamentales de la sociedad: espacio y tiempo. Respecto al control del espacio, Castells expone que en la "sociedad red" están surgiendo dos lógicas espaciales, la del espacio de los flujos y la del espacio de los lugares. El espacio de los flujos:

organiza la simultaneidad de las prácticas sociales a distancia, por medio de las telecomunicaciones y los sistemas de información. El espacio de los lugares privilegia la interacción social y la organización institucional atendiendo a la contigüidad física. Lo que distingue a la nueva estructura social, la sociedad red, es que la mayoría de los procesos dominantes, que concentran poder, riqueza e información, se organizan en el espacio de los flujos. La mayor parte de la experiencia y el sentido humanos siguen teniendo una base local. La disyunción entre las dos lógicas espaciales es un mecanismo fundamental de dominio en nuestras sociedades porque desplaza el núcleo de los procesos económicos, simbólicos y políticos del ámbito donde puede construirse el sentido social y puede ejercerse control político. Así pues, el hincapié de los ecologistas en la localidad y en el control de la gente de sus espacios vitales es un reto a una palanca básica del nuevo sistema de poder. (Castells, 2001: 149)

Respecto al control del tiempo, Castells indica que el movimiento ecologista es, probablemente, el actor más importante en la proyección de una temporalidad nueva y revolucionaria, y especifica que existen tres formas de temporalidad: el tiempo de reloj, el tiempo atemporal y el tiempo gracial. El tiempo de reloj, característico del industrialismo, se caracteriza por la secuencia cronológica de los acontecimientos y por la disciplina de la conducta humana a un horario predeterminado. El tiempo atemporal, que caracteriza a los procesos dominantes de la sociedad red, se da cuando las características de un contexto determinado provocan una perturbación sistémica en el orden secuencial realizados en el paradigma informacional y la sociedad red. El tiempo glacial implica que la relación entre los humanos es a muy largo plazo y evolutiva, por ello Castells propone la idea de que el movimiento ecologista se caracteriza precisamente por el proyecto de introducir una perspectiva de tiempo glacial en nuestra temporalidad, tanto en cuanto a la conciencia como a la política. El pensamiento ecológico considera, así, la interacción de todas las formas de la materia en una perspectiva evolucionista. La idea de limitar el uso de los recursos a los de carácter renovable se efectúa basándose en la noción de que la alteración del equilibrio básico del planeta y del universo puede, con el tiempo, deshacer el delicado equilibrio ecológico, con consecuencias catastróficas. 
Otra propuesta de clasificación, apoyándonos en Brand (2000), sería distinguir cuatro estrategias teóricas de desarrollo sostenible: 1) Business as usual. Esta estrategia es asumida por el enfoque de la economía ambiental o enfoque de la sostenibilidad débil. Enfoque que opta por la incorporación del medio ambiente como variable dependiente, como factor de corrección, ya que más que una "ecologización de la economía" se pretende una "economía de la ecología" que sea capaz de incorporar los factores ambientales en términos de costes, dando valores monetarios a los recursos naturales en el cálculo contable (Pearce, 1976). 2) Modernización ecológica. Esta estrategia, que designa una perspectiva teórica sobre el cambio social en las sociedades industriales avanzadas, forma parte de un discurso dominante de la política medioambiental desarrollada en algunos países europeos desde finales de los años ochenta del siglo XX. Su objetivo es analizar y explicar las transformaciones sociales inducidas por el medio ambiente en las sociedades avanzadas (Mol, 1999; Mol y Spaargaren, 1993). 3) Ecologización estructural. Según esta estrategia, únicamente una modificación estructural del estilo de vida de Occidente, tanto en la producción como en las pautas de consumo, posibilitaría un desarrollo sostenible. Un tipo de desarrollo que incorpora las fuertes dosis de irracionalidad que conlleva la metodología subyacente del conocimiento. Básicamente, esta estrategia, cuyo máximo exponente es Daly (1991), asume en todas sus consecuencias el principio de entropía y enfatiza la incompatibilidad entre el crecimiento económico y la sostenibilidad, al tener el mundo de lo físico sus límites que le impiden crecer indefinidamente. 4) Tradicionalismo antimaterialista. Para esta estrategia discursiva la moderna civilización occidental y su relación instrumental con la naturaleza se presentan como las causas del mal. Ante este panorama, se propone la incorporación de la dimensión moral al discurso económico dominante, ya que o cambiamos de rumbo y de fundamentos ideológicos o nos dirigimos hacia la locura de la insaciable ambición y la orgía de la envidia (Schumacher, 1973; Etzioni, 1988).

\section{Desarrollo humano}

El surgimiento de los derechos de solidaridad (económicos, sociales y culturales), en general, y del derecho al desarrollo, en particular, tiene que ver con el enfoque de los derechos humanos, que figura en el artículo 28 de la Declaración Universal de los Derechos Humanos. Desde este enfoque, y desde la perspectiva de la justicia como imparcialidad (Rawls, 1996; Nussbaum, 1998), se evalúa el desarrollo y la equidad en un espacio más amplio que el 
de los ingresos, un espacio que no es sólo la propia satisfacción o utilidad, ni sólo la existencia de reglas iguales y comunes, sino el de las posibilidades para elegir y obtener la vida que tenemos razones para valorar (Sen, 1999; Nussbaum, 2002). En este sentido, y desde la preocupación por promocionar los derechos de solidaridad, emerge la estrategia de desarrollo humano. Es decir, se asume que la Declaración Universal de los Derechos Humanos se refiere específicamente a la necesidad de una realización efectiva del derecho al desarrollo humano.

Por tanto, una variante del derecho al desarrollo es la estrategia de desarrollo humano, que es especificada originariamente por el denominado "enfoque de las necesidades básicas". Este enfoque pretendía alcanzar la satisfacción universal de las necesidades básicas, definidas como las que garantizaban un nivel de vida mínimo que toda sociedad debería establecer para los grupos más pobres de sus habitantes. Este enfoque tenía tres antecedentes teóricos: 1) el discurso anual de McNamara ante el consejo de Gobernadores del Banco Mundial de 1972, donde planteaba que, como las poblaciones de los países del Sur no son capaces de asumir su propio destino y de satisfacer sus necesidades más esenciales, los gobiernos de los países en desarrollo debían preocuparse más de las necesidades humanas esenciales; 2) la Declaración de Cocoyoc (1974, México), integrada en gran parte por intelectuales miembros del Foro del Tercer Mundo y; 3) el informe ¿Qué hacer? de la fundación Hammarskjöld. Este informe, publicado en 1975, intentaba plantear un "desarrollo distinto" basado en la satisfacción de las necesidades, la self-reliance o desarrollo autónomo, la armonía con la naturaleza y los cambios estructurales (Rist, 2002).

En 1975, la Conferencia Mundial sobre el Empleo de la Organización Internacional del Trabajo (OIT) definió las siguientes cuatro categorías de necesidades básicas: 1) el consumo alimentario, la vivienda y el vestido para tener un nivel de vida mínimamente digno; 2) el acceso a servicios públicos de educación, sanidad, transporte, agua potable y alcantarillado; 3) la posibilidad de tener un empleo adecuadamente remunerado y; 4) el derecho a participar en las decisiones que afectan a la forma de vida de la gente y a vivir en un medio ambiente sano, humano y satisfactorio. Adicionalmente, la conferencia de la OIT de 1975 aprobó un programa de acción que se centraba en los dos primeros tipos de necesidades básicas. Inspirado, entre otros por L. Emmerij, "quien luego sería presidente del Centro de Desarrollo de la OCDE, el documento de la Conferencia proponía satisfacer las necesidades básicas antes de finales de siglo con medidas como: la redistribución de la inversión y de la propiedad o del uso de la tierra, y la organización de los trabajadores rurales en sindicatos u otras formas de defensa colectiva" (Bustelo, 1998: 153). 
El concepto de "desarrollo distinto" fue incluido en la UNESCO en el informe MacBride de 1980. Esta institución entendía que en una sociedad heterogénea culturalmente no podía haber un concepto unívoco de desarrollo, por lo que se debía buscar una idea de desarrollo que fuera lo suficientemente moldeable, asimilable a cada una de las culturas, sin perder los objetivos comunes de mejorar las condiciones de vida de la sociedad, avanzar en la lucha contra la pobreza, etc. Por ello en la conferencia sobre políticas culturales que tuvo lugar en México, D. F., en 1982, se planteó la dimensión cultural del desarrollo, estableciéndose que, ante la diversidad social y cultural, el único común denominador es el "hombre" y, por tanto, el "hombre" debe ser el centro de análisis de ese largo proceso que es el desarrollo. La Resolución 41/128, adoptada por la Asamblea General de Naciones Unidas el 4 de diciembre de 1986, planteaba, también, un concepto de desarrollo situado en el origen y el fin de la actividad humana, pues "el hombre debe buscar y lograr ese derecho". Por su parte, el Programa de Naciones Unidas para el Desarrollo (PNUD) publicó en 1990 el Primer Informe sobre el Desarrollo Humano.

El Primer Informe sobre el Desarrollo Humano introdujo el índice de desarrollo humano (IDH) con el fin de utilizar unos indicadores menos dependientes de unas variables exclusivamente económicas y más adecuados para evaluar el desarrollo humano. El índice se basaba en datos nacionales y combinaba la esperanza de vida, las tasas de alfabetización de la población adulta, las tasas de matriculación en la enseñanza primaria, secundaria y superior, y el PIB real per cápita. Sobre este conjunto de variables, la denominada perspectiva sobre género y desarrollo ha destacado la centralidad de la variable género tanto para el cambio económico y social como para el desarrollo. La reflexión sobre cómo las mujeres participan en los procesos de desarrollo ha evolucionado desde una posición que considera las "mujeres en el desarrollo" a otra orientada a pensar en el "género en el desarrollo". "Mujeres en el desarrollo" y "género en el desarrollo" son dos maneras diferentes de percibir y abordar el tema de la subordinación y el trabajo de las mujeres en los modelos y estrategias globales de desarrollo. Para "mujeres en el desarro1lo", el problema está en la exclusión de las mujeres del proceso de desarrollo, y el objetivo que pretende es lograr un desarrollo más eficiente. Sin embargo, este enfoque ha aumentado a menudo la carga de trabajo de las mujeres sin lograr un mayor poder económico. Las mujeres no han sido consultadas sobre el tipo de desarrollo e integración que buscaban. Se da una "integración" en el mundo de los hombres sin un cambio en las relaciones de poder. Para "género en el desarrollo", el problema se encuentra en las relaciones desiguales de poder que frenan un desarrollo igualitario y la plena participación de mujeres, y su objetivo es un desarrollo sostenible e igualitario con 
toma de decisiones compartidas entre mujeres y hombres. Esta orientación se puede entender como un esfuerzo para mejorar la posición de las mujeres en relación con los hombres de manera que beneficie y transforme a la sociedad en su totalidad.

Las conferencias mundiales organizadas por las Naciones Unidas - desde la de México, en 1975, pasando por las de Copenhague, en 1980, Nairobi, en 1985, El Cairo, en 1994, hasta la de Beijing, en 1995- han actuado como un poderoso mecanismo para debatir las preocupaciones de las mujeres y establecer programas a escala mundial. Además, actualmente, "prácticamente todos los organismos han incorporado análisis y programas con perspectiva de género de algún modo relacionado con su ámbito específico de trabajo. Este esfuerzo se ha designado como 'integración del género en el trabajo central' (gender mainstreaming) y ha requerido una mayor representación de las mujeres en todos los niveles así como una mayor integración de los temas de género en sus actividades". En este sentido, el informe sobre Desarrollo Humano de 1995 amplió su trabajo sobre indicadores de desarrollo humano con el fin de incluir dos índices nacionales complementarios: el índice de desarrollo relativo al género (IDG) — basado en las diferencias de género en relación con la esperanza de vida, los ingresos obtenidos a través del trabajo, el analfabetismo y la matriculación en la enseñanza - y el índice de potenciación de género (IPG), basado en la proporción de mujeres parlamentarias y de las que ocupan puestos en la administración pública y la dirección de empresas, así como también en los estamentos profesionales y técnicos.

Durante la década de los noventa, bajo la atención preferente al "desarrollo humano", se configura "un nuevo humanitarismo" que se mueve con pautas que comprenden lo que se ha venido a llamar Tercera Ola democratizadora, y ello en un contexto en el que se generaliza la idea de que el papel de los Estados ha cambiado a consecuencia del surgimiento de una sociedad red en la que el Estado cumple funciones de articulación e integración hacia adentro y hacia fuera, y en la que también las instituciones no estatales y las empresas han de asumir responsabilidades por el desarrollo. Un desarrollo enmarcado y conducido por un "buen gobierno" capaz de asegurar su gobernabilidad democrática y la formación progresiva de una comunidad internacional basada en la justicia. En este sentido, en la Tercera Ola democratizadora se ha aumentado enormemente el número de países que se han transformado en regímenes políticos democráticos, y los países que no lo han hecho se ven forzados a adoptar principios de legitimidad democrática promovidos por unas organizaciones internacionales que han aceptado incorporar como proyecto normativo la implantación del denominado régimen de derechos humanos. Régimen que se apoya en una filosofía y en un sistema de derecho 
internacional impulsado por un conglomerado de instituciones y organizaciones internacionales y transnacionales diversas, ya sea que tengan un carácter formal como la ONU o diversas organizaciones regionales y sectoriales, o bien se trate de asociaciones de carácter informal. Para esta filosofía el mundo se muestra cada vez más como un hábitat colectivo de los humanos que impone regulaciones obligatorias para todos, que deberían estar orientadas a los derechos humanos y a la justicia y no a los intereses generales del hegemonismo o de sus adláteres.

La progresiva aceptación del régimen de los derechos humanos ha implicado una problematización de la seguridad y la reproblematización del subdesarrollo como un foco de peligro. El desarrollo se contempla como un asunto que no puede abandonarse a su suerte, ya que las amenazas a la seguridad del Norte ya no se interpretan sólo como conflictos interestatales que tienen que analizarse en clave de disuasión nuclear y de política de alianzas. En todo caso, el desarrollo como ayuda humanitaria es contemplado como un instrumento de regulación internacional y justificado en tanto que la interdependencia creciente:

ha hecho emerger verdaderos "bienes públicos globales", ¿cómo podremos construir la gobernanza necesaria para asegurar su provisión efectiva? Esto no es ninguna cuestión técnica: sin la provisión de esos bienes no hay gobernabilidad; volvemos al estado de naturaleza al que están retrotrayéndose tantos Estados fracasados y en riesgo que, en un mundo interdependiente, amenazan irremediablemente la seguridad de todos. (Prats, 2005: 33)

Esta provisión requiere de un sistema de "gobernanza" o "buen gobierno" en el que converjan desarrollo y seguridad. Según Duffield (2004: 69), el nuevo discurso de la seguridad ve que "el subdesarrollo es peligroso ya que puede conducir a la violencia; y, por su parte, el conflicto consolida y profundiza ese peligro. Las sociedades empeoran su situación y, además, son más propensas a que se produzcan estallidos de inestabilidad. Estos sentimientos comúnmente aceptados han proporcionado una base racional para la incorporación generalizada de un compromiso con las actividades de prevención de conflictos y de resolución de conflictos como parte de la política de ayuda oficial desde mediados de los noventa". Esto denota un cambio importante que hace que la política oficial de donaciones se convierta en intervencionismo, y que está estrechamente asociado con la seguridad. La política del desarrollo no sólo ha sido radicalizada para hacer frente a esta situación, sino que, significativamente, refleja un nuevo espacio de actuación de la seguridad en la que el subdesarrollo se considera un peligro. Al refor- 
zarse mutuamente las nociones de desarrollo y seguridad, conseguir una se considera esencial para poder conseguir la otra. El desarrollo es en última instancia imposible sin estabilidad, y, al mismo tiempo, la seguridad no se puede mantener sin desarrollo. Esta convergencia no es sólo una cuestión de normativa política. Tiene profundas implicaciones políticas y estructurales, y representa la creciente interacción entre actores militares y de seguridad, por un lado, y las organizaciones civiles y las no gubernamentales, por el otro.

La lógica dominante del nuevo régimen de seguridad del Norte comparte, por tanto, el terreno del discurso del desarrollo, y plantea que la llamada gobernanza global o "buen gobierno global" se impone a consecuencia de que la cada vez mayor interconexión del sistema mundial ha magnificado la amenaza de la internacionalización de la inestabilidad del Sur. Esto se refiere a los flujos de refugiados, pero también a la aclamada capacidad para trastornar las actividades comerciales y, mediante el apoyo a la expansión de redes relacionadas con el terrorismo y otras actividades criminales, a la posibilidad de tener un impacto más directo en el Norte. En este sentido, la guerra ha resultado, señala Duffield (2004), apropiada para reproblematizar la seguridad presentando el subdesarrollo como un peligro, y también para, mediante su radicalización, reinventar el papel del desarrollo. Con este proceso de redescubrimiento del conflicto, y al tratar de dar respuesta a sus nuevos desafíos, a pesar de medio siglo de fracasos el desarrollo ha resurgido fortalecido. En concreto, ha supuesto que se representen las nuevas guerras de modo que parezcan receptivas a una asistencia al desarrollo gestionada adecuadamente.

La asociación subdesarrollo y conflicto ha supuesto, en definitiva, considerar el conflicto como el resultado de una enfermedad endógena al desarrollo del Sur. Desde esta perspectiva, la reinvención del desarrollo, en la década de los noventa, como una forma de prevención de conflictos, es una parte importante de la radicalización de las políticas desarrollistas. La idea de que el conflicto destroza el desarrollo y profundiza la pobreza represeta un papel importante a la hora de movilizar los complejos estratégicos de la paz liberal. Estas políticas desarrollistas han generado un nuevo "ethos desarrollista" vinculado a la sustitución del bloque bipolar Este-Oeste por el modelo bipolar Norte-Sur, países ricos frente a países pobres. El "ethos desarrollista" ha seguido dos direcciones claramente distintas. En el Norte, se exige el redesarrollo, es decir, desarrollar de nuevo lo que se había desarrollado mal o resultaba ya obsoleto. En el Sur, el redesarrollo exige también desmantelar lo que había quedado del "proceso de ajuste" de la década de 1980. La obsesión por la competitividad, el temor a ser dejado fuera de la carrera, compele a aceptar la destrucción de secciones completas de lo que fue 
"desarrollado" en los últimos 30 años. Sacrificado en el altar del redesarro1lo, se le insertará en diseños transnacionales congruentes con la demanda del mercado mundial, y con la necesidad de implementar una gobernanza global, acorde con los nuevos procesos de división internacional del trabajo.

Estos procesos implican un reparto de naturaleza dual de la producción mundial, concentrando en el Norte las tareas intensivas en educación avanzada, ciencia y tecnología, mientras que se despliegan por el Sur gran parte de las labores productivas con baja participación de actividades locales intensivas en conocimiento. En este sentido, el Sur vive una reinserción neoperiférica en la economía internacional. Reinserción teorizada por Wallerstein (1987) en su teoría del sistema mundial y en la que la economía-mundo se caracteriza por un ritmo desigual del desarrollo y, por tanto, retiene la constitución asimétrica, muy desigualitaria, del sistema, con partes altamente desarrolladas, menos desarrolladas y relativamente subdesarrolladas.

\section{Crecimiento y desarrollo endógeno}

El paradigma del desarrollo endógeno no descuida los contextos territoriales, institucionales y sociales al ser la historia y geografía de un país las que canalizan hacia un cierto ámbito de la división internacional del trabajo y las que determinan, al mismo tiempo, el diseño territorial. Esto significa, por el papel central que otorga el entorno institucional local, considerar el desarrollo industrial de otra forma, y de atribuir a la historia económica un nuevo y más relevante papel: descubrir la identidad de los lugares y de las comunidades para explicar tiempos y modos del arraigo de los procesos de industrialización. Ante todo, se trata de ver el mundo de la industria no sólo como una red de interdependencias técnicas entre fábricas, del intercambio entre empresas, sino como un calidoscopio de sistemas productivos locales interconectados. Como exponen Becattini (2006) y Guenzi (2006), todo lo que puede hacer una gran empresa en términos de eficiencia puede ser realizado no por una empresa individual, sino por una población de empresas especializadas que operan en un contexto adecuado de nexos sociales, culturales e institucionales, y que constituyen el capital social de un entorno local/regional (Fernández, Amin y Vigil, 2008).

Esta aproximación territorial al desarrollo se apoya en los aportes de una diversidad de corrientes teóricas nucleadas en torno a: 1) la especialización flexible, los distritos industriales, los medios innovadores y la Nueva Geografía Económica; 2) los clusters industriales de Porter (1990), las regiones de aprendizaje y los sistemas regionales de innovación. Cada una de estas 
corrientes ha desarrollado aspectos específicos a partir de los cuales se destaca el papel de las localidades y de las regiones como epicentros para comprender los procesos de desarrollo (Brunet y Böcker, 2013). De ahí que la teoría del desarrollo endógeno tiene que ver con el redescubrimiento de la particularidad regional o local como política de desarrollo autónomo o self-reliance. Para ello se apoya en la idea de que al tomar en cuenta las singularidades socioeconómicas, políticas y culturales de cada región, podrá surgir endógenamente el desarrollo o, por lo menos, un impulso que lleve en esa dirección, confirmándose así que, por su propia definición, el concepto de desarrollo refiere a un cambio inmanente, endógeno, generado desde dentro del territorio, y que opera por medio del despliegue de potencialidades inherentes (Storper, 1997; Keating, 1998). Esta estrategia de desarrollo se produce en un contexto en el que los gobiernos nacionales dejan de ser los actores estratégicos en las políticas de desarrollo, gobiernos que ya no pretenden asumir toda la responsabilidad por el desarrollo nacional, ya que se parte de la creencia de que los desafíos específicos del desarrollo se dan, cada vez más, en el espacio metropolitano y regional. La movilización de energías colectivas a este nivel se consigue mediante la construcción de espacios públicos democráticos regionales o metropolitanos, que acaben generando identidades y comunidades que es preciso saber articular dentro del Estado nacional. Así, si durante las décadas de los cincuenta y sesenta las políticas de desarrollo regional tenían como actor estratégico al Estado nacional, tal y como prescribía la teoría de los polos de crecimiento, en las décadas de 1980 y 1990 se configurará una estrategia de desarrollo alternativo basado en actores, recursos y capacidades locales y regionales, la cual Sachs (1974) denominó "ecodesarrollo", basado en la geografía humana de las diferencias y en la especificidad territorial. Estrategia que se apoya en un esquema analítico que deposita la obtención de competitividad a nivel global en las calidades internas de localidades y regiones (a partir de la acción conjunta e interactiva de actores institucionales y económicos a nivel territorial).

Esta estrategia va a provocar en la década de los noventa, por la crisis de la deuda y las políticas de ajuste, un giro en los estudios neoclásicos sobre el desarrollo, originados en el seno del Banco Mundial (1991, 1993). Ese giro fue el resultado tanto del éxito de los "dragones asiáticos" (Corea del Sur y Taiwán, entre otros), como por el cada vez mayor escepticismo sobre la bondad de las recetas neoclásicas propugnadas en la década de los ochenta. Sin embargo, se trató de un enfoque favorable al mercado o que armonizase con éste, pues se justificaba la intervención estatal sólo en el caso de que el mercado estuviera insuficientemente desarrollado o si su libre funcionamiento fuera insuficiente. Admite, asimismo, la necesidad de políticas deliberadas 
para mantener la estabilidad macroeconómica, crear un entorno competitivo para las empresas, efectuar inversiones en infraestructuras y en educación y sanidad y potenciar el desarrollo institucional para un mejoramiento de la eficacia de la administración pública.

Esta propuesta de "desarrollo desde dentro" implicará, por un lado, evaluar el papel de las grandes empresas en los procesos de desarrollo endógeno, y, por otro, considerar que las políticas de desarrollo no pueden ser exclusivamente externas o internas, sino que deben tomar en cuenta ambos factores, así como la posición económica de los sistemas regionales de producción dentro de un contexto global. Por ejemplo, esta propuesta será retomada por el neoestructuralismo latinoamericano en la década de los noventa (CEPAL, 1994). Para los neoestructuralistas la equidad favorece el crecimiento y refuerza la competitividad auténtica. Una competitividad no basada en la sobreexplotación de los recursos naturales o en bajos salarios. De este modo, en el documento "Transformación productiva con equidad" se ponía el énfasis en la competitividad vía progreso técnico y se introducía la necesidad de una aproximación integral (social, institucional, política, etc.) al desarrollo, incorporando a éste la dimensión territorial y medioambiental.

Sunkel (1991), y Sunkel y Zuleta (1990), propondrán un modelo de desarrollo desde dentro, en torno a un proceso endógeno de acumulación y de generación de progreso técnico donde el Estado es decisivo en la orientación y puesta en marcha de políticas productivas, agrícolas, medioambientales y de tecnología e innovación. Se trata de dar un nuevo impulso a la industrialización, de buscar, retomar y superar

el desafío industrializador original de Prebisch en torno a generar un proceso endógeno de acumulación y de absorción y generación de progreso técnico -incluso por medio de la inversión privada extranjera - que origine una capacidad de decisión propia de crecer con dinamismo. Tal concepción estratégica no está orientada, a priori, a favorecer la sustitución de importaciones, lo cual finalmente llevaría a un callejón sin salida. Por el contrario, en esta propuesta se dejan abiertas las opciones para orientar la industrialización desde dentro hacia los mercados internos y externos que se consideran prioritarios y prometedores en la estrategia de desarrollo a largo plazo. Sunkel y Zuleta (1990: 43)

La atención hacia el papel que juegan los grupos industriales y las inversiones procedentes del exterior en el desarrollo económico, es consecuencia de la constatación (e interpretación) de que los cambios en la organización de las grandes empresas facilitan las estrategias de desarrollo de los territorios. De ahí que se trate de fomentar las inversiones externas en las nuevas políticas regionales de desarrollo. Es decir, se plantea abordar el desarrollo 
endógeno con políticas de incentivos a la localización de grandes empresas, debido a que éstas están adoptando formas de organización más flexibles que favorecen la integración de las unidades de empresas externas en los sistemas productivos locales.

La literatura económica sobre desarrollo endógeno parte de los modelos de crecimiento endógeno (Romer, 1986; 1990), que tratan de acomodar a la realidad los modelos neoclásicos de crecimiento. Lo que se conoce como modelos de crecimiento endógeno y/o teoría del crecimiento endógeno empezaron a ganar terreno en la segunda mitad de la década de 1980. Esta nueva teoría relaja varias de las hipótesis del modelo neoclásico exógeno para dar protagonismo a factores relevantes en la determinación del crecimiento económico que hasta ahora no se habían tratado adecuadamente. Pero, en primer lugar, esta nueva teoría pretendía dar respuesta a las debilidades de la teoría neoclásica, cuyas hipótesis eran acusadas de simplificar en exceso y, con ello, condicionar sus resultados. Se observaba, por ejemplo, que los mercados no suelen observar las condiciones de competencia perfecta, pues existen rendimientos crecientes de escala e información asimétrica. Además, el sector privado no es capaz de producir todos los bienes y servicios demandados en una sociedad: existen bienes públicos y se producen externalidades como consecuencia de la producción o consumo de ciertos bienes. Por otro lado, el proceso de innovación tecnológica no es siempre un factor exógeno generado fuera del mercado. Muchos de los descubrimientos y avances que han condicionado la producción durante el pasado siglo, y que han incrementado el nivel de vida de la población, se generaron en el seno del mercado, llevados a cabo por empresas que trataban de conseguir beneficios.

De este modo, con la teoría del crecimiento endógeno se abandona la hipótesis de la competencia perfecta y se acepta que el avance tecnológico es un factor endógeno y que las rentas monopolistas condicionan los procesos de crecimiento. De esta forma al endogeneizar el avance tecnológico e incorporar el poder de mercado de las empresas en los procesos de innovación y crecimiento económico, los modelos de crecimiento endógeno han dado un nuevo impulso al crecimiento económico, apoyándose tanto en los modelos de derrame como en los modelos neoschumpeterianos. Los modelos de derrame conceden a las economías externas un papel estratégico en el crecimiento económico, ya que les permite introducir el concepto marshaliano de rendimientos crecientes y preservar, al mismo tiempo, el aparato analítico neoclásico. Se argumenta, al respecto, que toda inversión nueva produciría un efecto difusor, externo a la empresa que la realiza, que mejoraría la productividad de las empresas de la industria y podría ser de tal intensidad que 
permitiría mantener el crecimiento endógeno. El efecto derrame provendría de los diferentes tipos de inversión, en capital físico, en capital humano y en investigación y desarrollo.

Los modelos neoschumpeterianos conceden un papel importante a la innovación tecnológica. La innovación, para estos modelos, determina el funcionamiento del sistema económico como un proceso cíclico. El ciclo, para Schumpeter (1964), era la forma que asume el desarrollo económico en la era del capitalismo, y consideraba que cada ciclo era único, debido a la variedad de las innovaciones técnicas. Más aún, las tecnologías y las empresas, juntamente con los territorios, son los principales generadores de las posibilidades de producción del capitalismo contemporáneo. Un capitalismo, definido por Storper (1997), como una economía de aprendizaje, con el argumento de que el aprendizaje es el resultado competitivo del aumento de reflexividad.

Los modelos de crecimiento endógeno tienen las siguientes diferencias importantes con el modelo neoclásico: a) la economía crecería, al menos, a una tasa constante, sea cual sea el nivel de renta y capital, por lo que no existiría transición al estado estacionario; $b$ ) la tasa de crecimiento y el nivel de renta de la economía no estarían relacionados, por lo que los modelos de crecimiento endógeno no predicen la convergencia entre diversas economías $\mathrm{y} ; c)$ los impulsos externos que fomentan el aumento del ahorro o el avance tecnológico se transformarían en incrementos de la renta y favorecerían los procesos de crecimiento endógeno. Estas diferencias alejan a los modelos de crecimiento endógeno de los modelos neoclásicos y les acercan a las teorías del desarrollo endógeno. No se trata sólo de que las teorías del crecimiento y desarrollo endógeno compartan la visión, quizá optimista, de que los sistemas productivos reúnen un conjunto de factores materiales e inmateriales que permiten a las economías locales y regionales emprender vías diferenciadas de crecimiento que no necesariamente les llevan a la convergencia, y que, por tanto, existe un espacio para las políticas regionales e industriales. Los puntos de acuerdo van más allá. Para Vázquez (1999), las interpretaciones del crecimiento y desarrollo endógeno coinciden en que cuando se analiza la dinámica de las economías, lo prioritario es identificar los factores y los mecanismos que favorecen los procesos de crecimiento y cambio estructural y no si existe o no convergencia entre las economías.

Por su parte, hay que destacar que los modelos de crecimiento endógeno rechazan la necesidad o la posibilidad de imitar mecánicamente a las sociedades industriales, tal y como prescribían las teorías de la modernización como la crítica a la misma realizada desde las teorías del intercambio desigual y de la dependencia. A pesar de sus diferencias, las anteriores teorías 
compartían una serie de asunciones sobre el desarrollo que en las teorías del desarrollo endógeno consideran que están en desuso (Becattini, 2006). Estas asunciones incluyen la idea de que el desarrollo debiera ser algo parecido a lo que eran los Estados Unidos y los países europeos, quizás incluyendo la Unión Soviética; la presunción de la existencia de una relación automática entre crecimiento económico y redistribución social; la idea de que el desarrollo es nacional, no regional ni local; que si había algún obstáculo, éste nunca sería de tipo natural; que antes o después se daría una igualación entre países y que, una vez alcanzado, el desarrollo era un estado permanente e irreversible.

Así, por ejemplo, la nueva geografía económica establece que el libre juego de las fuerzas del mercado conduce inexorablemente a una intensificación de las desigualdades regionales, tal como ya lo habían planteado Myrdal (1957). Esto es así porque en virtud de las economías de aglomeración, el crecimiento tiende a beneficiar acumulativamente a las economías de mayor desarrollo, en detrimento de las más atrasadas, dado que el capital humano, el conocimiento y la infraestructura son los factores más determinantes del crecimiento territorial, factores revalorizados por la revolución en la producción, información y tecnologías de la comunicación. Estas tecnologías permiten una gran expansión de la naturaleza de las esferas de control de las empresas, mercados e instituciones, lo que implica una retroalimentación más intensa e inmediata entre las diferentes partes de estas complejas estructuras, abaratamiento drástico de las diferentes formas de producción material e incrementos significativos en la diversidad de inputs y outputs materiales e intangibles. Además, se ha dado una amplísima extensión espacial y profundización social de la lógica de las relaciones de mercado, en parte facilitada por el salto tecnológico (especialmente por el abaratamiento de las telecomunicaciones y los medios de comunicación como vehículos de las relaciones de mercado, y a través de la extensión de la infraestructura física) (Storper, 1990; 1997).

En el campo del desarrollo territorial se establece, por tanto, la idea de que el desarrollo endógeno está íntimamente articulado a los procesos de globalización económica, en un movimiento dialéctico y mutuamente constitutivo (Bagnasco, 2007). En esto consiste la denominada interrelación entre fuerzas globales y condiciones locales, y que produce resultados específicos en términos de desarrollo, y para Storper (1990: 15), de reflexibilidad económica. Este último término hace referencia a la posibilidad, para grupos de agentes de las diferentes esferas del capitalismo moderno - empresas, mercados, gobiernos, economías domésticas u otros colectivos-, de dar forma al curso de la evolución económica. Éstos pueden hacerlo porque ahora 
"pueden reflexionar sobre el funcionamiento de sus entornos respectivos de una forma que no está limitada por los parámetros existentes, y donde ciertos grupos están explícitamente interesados en reorganizar dichos entornos en beneficio propio (innovación)". Uno de los efectos de este tipo de acción es la transformación de los roles de ciudades y regiones, ya que la globalización al reestructurar el territorio cambia el concepto de ciudad y de región. Este cambio que tiene que ver con el hecho de que las ciudades y regiones se definen por la posición que ocupan en las estructuras reticulares de ciudades y territorios en las que se insertan.

\section{A modo de conclusión}

La principal conclusión que puede extraerse de estas páginas es que la noción de desarrollo sólo puede entenderse como una construcción social que debe ser interpretada en su contexto histórico. Así, el dominio neoclásico, que marca el capitalismo globalizado y financiarizado de las últimas décadas del siglo XX e inicios del siglo XXI, tuvo lugar a consecuencia de que el crecimiento "dorado" — de 1950 y 1960 - se vio bruscamente alterado en los primeros años de los setenta. Esta alteración apoyó la creencia de que ya no se daban las condiciones para una expansión rentable utilizando métodos keynesianos. Ello ha llevado a economistas y gobiernos al siguiente convencimiento: puesto que las políticas de demanda — monetaria y fiscal— tenían a largo plazo efectos inapreciables y difíciles de predecir, el objetivo de la política económica debería concentrarse en favorecer el funcionamiento eficiente de los mercados y crear las condiciones de estabilidad que favorecieran el crecimiento sostenido de la economía. Esto trajo consigo la dispersión de las conceptualizaciones sobre el desarrollo, que ha tenido lugar simultáneamente a la configuración del proceso denominado "globalismo" (Beck, 1998), esto es, la integración económica mundial bajo el dominio de una práctica e ideología económica, social y política neoliberal o neoclásica. Práctica e ideología que incorpora lo que se ha denominado el "evangelio de la competencia".

Un evangelio para el que la competencia es la respuesta a casi todos los problemas económicos. Si empeora el problema del desempleo, entonces es necesario aumentar la competitividad. Si existe un problema creciente de pobreza en algunos países, entonces hay que ser más competitivo. Asimismo, la educación y la capacitación deben orientarse más a la panacea de la competencia. Como resultado, las sociedades se ven progresivamente implicadas en una batalla económica despiadada (Emmerij, 1998). Es el 
valor de la acumulación competitiva, juntamente con el valor de progreso, el que guía el pensamiento y la acción de la planificación y desarrollo, y en relación al cual determinados agentes económicos e institucionales orientan sus expectativas y acciones.

Recibido: octubre de 2013

Revisado: mayo de 2014

Correspondencia: Universitat Rovira i Virgili/Av. Universitat/1 (43204)/ Reus/España/correo electrónico: IBI, ignasi.brunet@urv.cat; RBZ, rafael. zavaro@gmail.com

\section{Bibliografía}

Arocena, Rodrigo y Judith Sutz (2000), La universidad latinoamericana del futuro, México, Unión de Universidades de América Latina.

Bagnasco, Arnaldo (2007), "El capitalismo que cambia, el trabajo y las condiciones de vida", Sociología del Trabajo, núm. 61, pp. 7-29.

Banco Mundial (1993), The East Asian Miracle. Economic Growth and Public Policy, Nueva York, Oxford University Press.

Banco Mundial (1991), Informe sobre el desarrollo mundial 1991, Washington, Banco Mundial.

Becattini, Giacomo (2006), "Vicisitudes y potencialidades de un concepto: el distrito industrial”, Economía Industrial, núm. 359.

Beck, Ulrich (1998), ¿Qué es la globalización? Falacias del globalismo, respuestas a la globalización, Barcelona, Paidós.

Brand, Kai Werner (2000), "Del debate sobre el desarrollo sostenible a las políticas medioambientales", en Iñaki Bárcena, Pedro Urbana y Mario Zubiaga (eds.), Desarrollo sostenible: un concepto polémico, Bilbao, Universidad del País Vasco.

Brunet, Ignasi y Rafael Böcker (2013), Capitalismo global: aspectos sociológicos, Madrid, Grupo 5.

Bustelo, Pablo (1998), Teorías contemporáneas del desarrollo económico, Madrid, Síntesis.

Camagni, Roberto (2005), Economía urbana, Barcelona, Antoni Bosch.

Castells, Manuel (2001), La era de la información. Vol. II. El poder de la identidad, México, Siglo XXI.

CEPAL (1994), El regionalismo abierto en América latina y el Caribe. La integración económica al servicio de la transformación productiva con equidad, Santiago, Naciones Unidas, CEPAL.

Daly, Herman E. (1991), "Economía ecológica y desarrollo sustentable", Documentación Social, núm. 89. 
Duffield, Mark (2004), Las nuevas guerras en el mundo global. La convergencia entre desarrollo y seguridad, Madrid, Libros de la Catarata.

Emmerij, Louis (1998), "Teoría y práctica del desarrollo: ensayo introductorio y conclusiones de política”, en Louis Emmerij y José Núñez del Arco (comps.), El desarrollo económico y social en los umbrales del siglo XXI, Washington, Banco Interamericano de Desarrollo.

Etzioni, Amitai (1988), The Moral Dimension: toward a New Economics, Nueva York, The Free Press.

Fernández, Víctor Ramiro, Ash Amin y José Ignacio Vigil (comps.) (2008), Repensando el desarrollo regional. Contribuciones globales para una estrategia latinoamericana, Buenos Aires, Editorial Miño y Dávila.

Grupo de Lisboa (1995), Limits to Competition, Nueva York, MIT Press.

Guenzi, Alberto (2006), "El distrito industrial y su impacto en la historia económica", Economía Industrial, núm. 359.

Jameson, Fredric (2004), Una modernidad singular. Ensayo sobre la ontología del presente, Barcelona, Gedisa.

Jessop, Bob y Ngai-Ling Sum (2005), The Regulation Approach and beyond: Putting Capitalism Economies in threir Places, Londres, Edward Elgar.

Keating, Michael (1998), The New Regionalism in Western Europe. Territorial Restructuring and Political Change, Northampton, Edward Elgar.

Lélé, Sharachchandra M. (1991), "Sustainable Development: a Critical Review", World Development, vol. 19, núm. 6, pp. 607-621.

Meadows, Dennis H. (1972), Los limites del crecimiento: un informe del proyecto del Club de Roma sobre el predicamento de la humanidad, Madrid, Fondo de Cultura Económica.

Mézáros, Itsván (2002), Para além do capital, Sâo Paulo, Editora da UNICAMP, Boitempo Editorial.

Mol, Arthur P. J. (1999), "Ecological Modernization and the Environmental Transition of Europe: between National Variations and Common Denominators", Journal of Environmental Policy and Planning, núm. 1.

Mol, Arthur P. J. y Gert Spaargaren (1993), "Environment, Modernity and the Risksociety: the Apocalyptic Horizon of Environmental Reform”, International Sociology, vol. 4, núm. 8.

Myrdal, Gunnar (1957), The Self Organizing Economy, Cambridge, Blackwell.

Naredo, José M. (2006), Raices económicas del deterioro ecológico y social. Más allá de los dogmas, Madrid, Siglo XXI.

Nussbaum, Marthe C. (2002), Las mujeres y el desarrollo humano, Barcelona, Herder.

Nussbaum, Marthe C. (1998), "Capacidades humanas y justicia social. En defensa del esencialismo aristotélico", en J. Riechmann (comp.), Necesitar, desear, vivir. Sobre necesidades, desarrollo humano, crecimiento económico y sustentabilidad, Madrid, Los Libros de la Catarata.

oCDE (2001), Innovative Clusters. Drivers of Nacional Innovation Systems, París, OCDE.

Pearce, David W. (1976), Enviromental Economics, Londres, Longman. 
Porter, Michael E. (1990), La ventaja competitiva de las naciones, Barcelona, Plaza y Janés.

Prats, Joan (2005), De la burocracia al management, del management a la gobernan$z a$, Madrid, Instituto Nacional de Administración Pública.

Rawls, John (1996), Liberalismo político, Barcelona, Crítica.

Rist, Gilbert (2002), El desarrollo: historia de una creencia occidental, Madrid, Libros de la Catarata.

Romer, Paul (1990), "Rendimientos crecientes y nuevos desarrollos en la teoría del crecimiento", Cuadernos Económicos del ICE, vol. 46, núm. 1, pp. 280-305.

Romer, Paul (1986), "Increasing Returns and Long-Run Growth", Journal of Political Economy, vol. 94, núm. 1, pp. 1002-1037.

Sachs, Ignacy (1974), "Ecodevelopment”, Ceres, vol. 15, núm. 4.

Schumacher, Erns (1973), Lo pequeño es hermoso, Barcelona, Crítica.

Schumpeter, J. (1964), Teoría del desarrollo económico, México, FCE.

Sempere, Joaquim y Jorge Riechmann (2000), Sociología y medio ambiente, Barcelona, Síntesis.

Sen, Amartya (1999), Desarrollo y libertad, Barcelona, Planeta.

Storper, Michael (1997), The Regional World: Territorial Development in a Global Economy, Nueva York, Guilford Press.

Storper, Michael (1990), "Industrialization and the Regional Question in the Third World: Lessons of Post-Imperialism; Prospects of Post-Fordism”, International Journal of Urban and Regional Research, vol. 14, núm. 3.

Sunkel, Osvaldo (1991), El desarrollo desde dentro. Un enfoque neoestructuralista para América Latina, México, FCE.

Sunkel, Osvaldo y Gustavo Zuleta (1990), "Neoestructuralismo versus neoliberalismo en los años noventa", Revista de la CEPAL, núm. 49.

Todaro, Michael P. (1982), Economía para un mundo en desarrollo, México, FCE.

Vázquez, Antonio (1999), Desarrollo, redes e innovación. Lecciones sobre el desarrollo endógeno, Madrid, Pirámide.

Wallerstein, Immanuel (1987), El moderno sistema mundial, México, Siglo XXI.

\section{Acerca de los autores}

Ignasi Brunet Icart es catedrático en el Área de Sociología de la Universitat Rovira i Virgili, España. Sus áreas de interés principales son teoría sociológica, sociología económica, sociología del trabajo y sociología de la educación. De sus publicaciones recientes citamos, en coautoría con R. Böcker, "Competitividad, competencias y fin del ciclo fordista", International Journal of Sociology of Education, vol. 3, núm. 1, 2014, pp. 1-25; y con A. Belzunegui y F. Valls, Pobreza y exclusión social de la juventud en España, Valencia, Tirant Humanidades, 2013. 
Rafael Böcker Zavaro es profesor en el Área de Sociología de la Universitat Rovira i Virgili, España. Sus áreas de interés principales son sociología económica, sociología histórica y política económica. Dos de sus publicaciones recientes son, en coautoría con I. Brunet, "Competitividad, competencias y fin del ciclo fordista", International Journal of Sociology of Education, vol. 3, núm. 1, 2014, pp. 1-25; y también con I. Brunet, Capitalismo global. Aspectos sociológicos, Madrid, Grupo 5, 2013. 
